\title{
Bioequivalence Study of Two Favipiravir Tablet Formulations in Healthy Male Subjects
}

\author{
Onursal Sağlam ${ }^{1}$, Gökçe Demiray ${ }^{1}$, Berrak Güney ${ }^{1}$, Emel Doğan-Kurtoğlu ${ }^{1}$, Merve \\ Ulusoy $^{1}$, Nihal Saraner ${ }^{1}$, Gamze Sevici ${ }^{1}$, Muradiye Nacak ${ }^{2}$, Aydın Erenmemişoğlu ${ }^{3}$, and \\ Vildan Tüzer ${ }^{4}$ \\ ${ }^{1}$ Novagenix Bioanalytical Drug R\&D Center \\ ${ }^{2}$ FARMAGEN-Good Clinical Practice Center \\ ${ }^{3}$ Alpan Farma R\&D Biotechnologies Ltd \\ ${ }^{4}$ Atabay Pharmaceuticals And Fine Chemicals Inc
}

September 24, 2020

\begin{abstract}
Abstract Background: As WHO expresses, COVID-19 is the infectious disease caused by the most recently discovered coronavirus. This new virus and disease were unknown before the outbreak began in Wuhan, China, in December 2019. COVID-19 is now a pandemic affecting many countries globally. Antiviral agents play fundamental role in Covid-19 treatment. Favipiravir is one of the favored agents and it still draws attention of generic drug industry which is constitutional for drug accessibility. Objective: The aim of this study is to demonstrate the bioequivalence of a new Favipiravir tablet formulation as compared to the reference tablet formulation in healthy male subjects under fasting conditions. To prove the bioequivalence, a randomised, single oral dose, cross-over, two-period study was carried out in 30 healthy subjects under fasting conditions. Plasma Favipiravir levels were quantified by using an in-house-developed high performance Liquid Chromatography Coupled to Tandem Mass Spectrometry (LC-MS/MS) method. Results: The 90\% CIs for the test/reference geometric mean ratios of the Cmax and AUC0-tlast were $92.92-119.89 \%$ and $94.00-99.77 \%$, respectively. Conclusions: This single-dose study has shown that the test and reference Favipiravir products met the required bioequivalence criteria. Besides, both products were well tolerated and safe. *The data that support the findings of this study are available from the corresponding author upon reasonable request. Some data may not be made available because of privacy or ethical restrictions.
\end{abstract}

\section{Introduction}

Favipiravir was approved in Japan for use in the event of an outbreak of novel or re-emerging influenza viral infections, where other influenza antiviral drugs are either not or insufficiently effective [1]. Favipiravir is a new antiviral drug against influenza. Favipravir is metabolized into favipiravir ribosyl triphosphate (favipiravir RTP) by an intracellular enzyme, and favipiravir RTP selectively inhibits RNA polymerase (RNA-dependent RNA polymerase) of the influenza virus, preventing replication of the influenza virus. The mechanism of action of favipiravir is the selective inhibition of RNA polymerase by favipiravir ribosyl triphosphate formed by cellular enzymes in the influenza virus leading to antiviral activity [2]. In an openlabel comparative controlled study of patients with Covid-19, those treated with favipiravir apeared to have faster viral clearance and higher rates of improvement in chest imaging, and in another prospective, randomized, controlled, open-label multicenter trial favipiravir significantly improved the latency to relief for pyrexia and cough [3,4].

The bioequivalence study is required for generic orally administered Favipiravir products by certain regulatory authorities $[5,6]$. Therefore, this study aims to compare the pharmacokinetic properties of a generic 
formulation to the reference product and to demonstrate the bioequivalence of the products with respect to the rate and extent of absorption of Favipiravir in healthy male subjects under fasting conditions.

\section{Subjects and Methods}

\section{Study population}

All subjects are adult males (aged 20-40 years) with normal weight according to the a body mass index BMI. The subjects who have atopic constitution or asthma and/or known allergy for Favipiravir and/or any of the excipients of the products and who have any history or presence of clinical relevance of cardiovascular, neurological, musculoskeletal, haematological, hepatic, gastrointestinal, renal, pulmonary, endocrinological, metabolism were excluded from the study. The inclusion and exclusion criteria were established clearly together with the reasons for withdrawal from the study. The subjects who were willing to participate in the clinical trial signed the informed consent form on their own freewill and understood that they could withdraw from the study anytime without specifying any reason.

\section{Study Design}

A single centre, open-label, randomised, single oral dose, cross-over, two-sequence, two-period study was conducted in 30 healthy, Caucasian, adult, male, human subjects under fasting conditions. This study was reviewed and approved by the Ethical Committee (Approval Date: 30.04.2020) and Turkish Medicines and Medical Devices Agency (Approval Date: 04.05.2020), and was held in Turkey according to the regulations run by Ministry of Health of the Republic of Turkey which are in compliance with Declaration of Helsinki and Good Clinical Principles (GCP) [7].

This study was conducted at FARMAGEN-Good Clinical Practice Center, Gaziantep, Turkey. The clinical study Spanned a period of approximately 9 days including prestudy screening, isolation period (5 days), wash-out period (48 hours) and final examination. The standard laboratory examinations in blood and urine were done consistent with the study protocol and the volunteers were checked for presence of HBsAg, HCV-Ab and HIV-Ab in serum. Also Covid-19 PCR tests were applied to the volunteers before isolation period and hospitalization. They were requested to provide a urine sample for a drug screen which include "amphetamines, cannabinoids, benzodiazepines, cocaine, opioids and barbiturates" and an alcohol breath test on entry examinations. All laboratory tests were carried out in a certified local laboratory.

Depending on the suitability of the volunteers clinical examination and laboratory results, isolation period was provided for 4 nights in single rooms reserved and it was important that the volunteers participating in the study do not come into contact with each other during the isolation and that the rules of isolation were followed due to Covid-19 Pandemic. After isolation period volunteers were transferred to vlinical center depending on negative Covid-19 PCR tests done once more.

A total of 30 subjects have been randomised and 29 subjects completed the clinical study. 10-hour fasted subjects were not allowed to drink water from $1 \mathrm{~h}$ before until $1 \mathrm{~h}$ after the administration of study products, except while dosing and they remained fasted until 4 hours after administration. Immediately after pre-dose sampling, 1 tablet of the test drug or 1 tablet of the reference drug (200 mg Favipiravir each case), were taken by the subjects with $240 \mathrm{~mL}$ of water. After the washout period (approximately 48 hours); in Period II, the subjects were administered the other drug they did not take in the Period I. The same procedures were applied in each period.

\section{Investigational medicinal products}

The test drug used was Favicovir $200 \mathrm{mg}$ Film Tablet, Atabay Kimya San. ve Tic. A.Ş.-Turkey (Batch No: FATA-P01; Expiration Date: 03.2022); the reference drug used was Avigan $200 \mathrm{mg}$ Film Tablet, Toyama Chemical Industry Co.Ltd.-Japan (Batch No: FG1881; Expiration Date: 07.2028).

\section{Blood Sampling and Study assessment}

The samples were drawn by a short intravenous catheter at pre-dose and at $0.17,0.25,0.50,0.75,1.00,1.33$, 
$1.66,2.00,2.50,3.00,3.50,4.00,5.00,6.00,8.00,10.00,14.00$, and 24.00 hours post-dose in each clinical study period, and they were collected into polypropylene tubes using $\mathrm{K}_{2}$ EDTA as anti-coagulating agent.

Volunteers were hospitalized at FARMAGEN-Good Clinical Practice Center after isolation period during the clinical study. An evening meal was provided at hospitalization days (total caloric value of approximately $1200 \mathrm{kcal}$ during study period. On medication days, a standard lunch (total caloric value is approximately $1200 \mathrm{kcal}$ ) was provided 4 hours after dosing, and a standard evening meal (total caloric value is approximately $1200 \mathrm{kcal}$ ) was provided 10 hours after dosing in each period Also standard light breakfast was provided in the evenings during hospitalization.

The blood samples were taken by a short intravenous catheter and they were collected into polypropylene tubes using $\mathrm{K}_{2}$ EDTA as anti-coagulating agent. After sampling, the samples were immediately refrigerated at approximately $2-8^{\circ} \mathrm{C}$ and will remain there for not more than 30 minutes. Following the centrifugation (3.000 rpm, $4-6^{\circ} \mathrm{C}, 10 \mathrm{~min}$ ), the separated plasma from each sample were transferred into two $3 \mathrm{~mL}$ transparent, polypropylene tubes. All the aliquoted plasma samples will be flash freezing immediately. The flash frozen samples (aliquoted plasma samples) were transferred to a deep-freezer and stored at $-70^{\circ} \mathrm{C}$ until they were transported to the bioanalytical center.

\section{Determination of Favipiravir Plasma Concentrations}

The bioanalytical phase of the study has been run at Novagenix Bioanalytical R\&D Center, Ankara, Turkey. In order to avoid bias, the analytical studies were operated as analytically blinded.

\section{Materials and methods}

\section{Chemicals}

Reference; Favipiravir, was supplied from Kohn\&Shawn Pharmatech Co., Ltd.,China., (Batch No: KSY3422-20200415) and Internal Standard; [13C15N] Favipiravir (IS), was supplied from AlsaChim, (MJ-ALS-20026-P2), France.

Solvents: Methanol and acetonitrile were supplied from Merck, Germany. Ultrapure (Type 1) Water was supplied from Milipore MilliQ Water Purification System; K2Edta Blank Human Plasma was supplied from Bioivt Laboratories International Ltd, UK.

\section{Instrument and conditions}

Shimadzu 8040 Tandem Mass Spectrometer (Shimadzu, Japan) was used equipped with electrospray ionization in the negative ion mode. Optimum seperation conditions were obtained with Shiseido Capcell PAK C18, $250 \times 4.6 \mathrm{~mm}, 5 \mu \mathrm{m}$ with mobile phase consisting of water and acetonitrile $(15 / 85, \mathrm{v} / \mathrm{v})$ with column oven temperature maintained at $30^{\circ} \mathrm{C}$. Flow rate was $1.2 \mathrm{~mL} / \mathrm{min}$. The multiple reaction monitoring (MRM) transitions were performed at m/z 156.1¿113.1and 158.1¿113for favipiravir and [13C15N] Favipiravir, simultaneously. Total run time for the method was $3.5 \mathrm{~min}$. The nebulizing gas and drying gas flow rates and the ESI voltage were $2.5 \mathrm{~L} / \mathrm{min}, 15 \mathrm{~L} / \mathrm{min}$ and $5500 \mathrm{~V}$, respectively. The gas used for nebulizing and drying was high pure nitrogen generated by Peak Scientific NL-60. Shimadzu LabSolutions Software version 5.93 was used for data acquisition and evaluation of chromatographic data.

\section{Preparation of standard and quality control (QC) samples}

Stock standard solutions of Favipiravir were prepared in methanol at a concentration of $5 \mathrm{mg} / \mathrm{mL}$. Working solutions in the concentration range of $1.6-600 \mu \mathrm{g} / \mathrm{mL}$ were prepared by diluting with methanol. The working IS was prepared in methanol at a concentration of $10 \mu \mathrm{g} / \mathrm{mL}$. Stock solutions of Favipiravir and IS were stored at $-20{ }^{\circ} \mathrm{C}$.

Calibration standards were prepared by spiking the appropriate amounts of standard solutions into blank plasma to obtain final concentration levels of $80,160,1000,5000,10000,20000,27000,30000 \mathrm{ng} / \mathrm{mL}$. The quality control samples were prepared similarly, at concentrations of 80,240, 1500, 12000 and $24000 \mathrm{ng} / \mathrm{mL}$. 
The lower limit of quantification (LLOQ), using $100 \mu \mathrm{L}$ human plasma, was $80 \mathrm{ng} / \mathrm{mL}$. Calibration standards and $\mathrm{QC}$ samples were stored at $-70^{*} \mathrm{C}$ freezer until analysis.

\section{Sample preparation}

The protein precipitation was the preferred choice of seperation because of the minimized steps in extraction of drug from matrix. Aliquots of $100 \mu \mathrm{L}$ plasma samples and $50 \mu \mathrm{L}$ of IS $(10 \mu \mathrm{g} / \mathrm{mL})$ was added into $10 \mathrm{~mL}$ centrifuge tube and vortexed for $5 \mathrm{~s}$. The mixture was precipitated with $500 \mu \mathrm{L}$ acetonitrile. After vortexing for $30 \mathrm{~s}$, the samples were centrifuged at $5500 \mathrm{rpm}$ for $10 \mathrm{~min}$. An aliquot of $5 \mu \mathrm{L}$ of the supernatant was injected into the LC-MS/MS system for analysis.

\section{Method validation}

The method validation was performed with $\mathrm{K}_{2}$ EDTA human plasma according to European Medicines Agency Guideline on Bioanalytical Method Validation [8]. The method was validated for selectivity, specificity, carry-over, linearity, precision and accuracy, recovery, dilution integrity, influence of haemolysed and hyperlipidaemic plasma, drug-drug interaction, matrix effect and stabilities.

The analytical curves were constructed from a blank sample (plasma sample processed without IS), a zero sample (plasma processed with IS) and eight concentrations of Favipiravir, including the LLOQ, ranging from 80 to $30000 \mathrm{ng} / \mathrm{mL}$. The concentrations were calculated using peak area ratios and the linearity of the calibration curve was determined using least squares regression analysis employing a weighted $(1 / \mathrm{x})$ linear $(\mathrm{y}=\mathrm{mx}+\mathrm{b})$ for Favipiravir. The acceptance criteria for each calculated standard concentration was not more than $15 \%$ deviation from the nominal value, except for the LLOQ which was set at $20 \%$. The within-batch precision and accuracy was evaluated by analyzing QC samples at five different concentration levels (80 $\mathrm{ng} / \mathrm{mL}$ (LLOQ), $240 \mathrm{ng} / \mathrm{mL}$ (QC Low), $1500 \mathrm{ng} / \mathrm{mL}$ (QC Medium), $12000 \mathrm{ng} / \mathrm{mL}(\mathrm{QC}$ High) and 24000 $\mathrm{ng} / \mathrm{mL}(\mathrm{ULLOQ})$ ) with six replicates in a batch. The between-batch precision and accuracy were determined by analyzing three different batches. The within-batch and between-batch values did not exceed $15 \%$ for QC samples, expected for LLOQ which did not exceed $20 \%$.

The selectivity was studied by checking the chromatograms obtained from eight different sources of human plasma including one haemolytic and one lipemic plasma. By comparing the chromatograms of those plasma samples spiked with Favipiravir and IS with the chromatograms of the blank plasma samples, no peak was found at the retention time of Favipiravir and IS in ten of the blank plasma samples. The recoveries were estimated by comparing the peak areas of Favipiravir in three replicates of QC samples with those of post-extraction blank matrix extracts at the corresponding concentrations. The matrix effects of Favipiravir were evaluated by comparing the peak areas of post-extraction blank plasma that were spiked at certain concentrations of $\mathrm{QC}$ samples with the areas obtained by the direct injection of the corresponding standard solutions. The stability of Favipiravir in the plasma samples was determined from three QC levels with six replicates each under the following conditions: Long-term stability at $-70^{\circ} \mathrm{C}$ for 60 days, short-term stability at RT for $5 \mathrm{~h}$, using processed samples in autosampler vials for $24 \mathrm{~h}$, and after four freeze/thaw cycles (-70 to RT).

An in-house high performance liquid chromatography with tandem mass spectrometry method (LC-MS/MS) was developed and validated to quantify Favipiravir in plasma.

The plasma samples were maintained at $-70^{\circ} \mathrm{C}$ during the assay. $0.1 \mathrm{~mL}$ of thawed samples to room temperature were transferred in a polypropylene tube and were prepared for analysis using protein precipitation according to SOP of bioanalytical center.

\section{Pharmacokinetic and Statistical Analyses}

According to only one literature, the intra-subject coefficient of variation (ISCV) for maximum plasma concentration $\left(\mathrm{C}_{\max }\right)$ was $17.0 \%$. Since there was insufficient information for ISCV, sample size was chosen as 30 subjects in order to demonstrate bioequivalence for a $2 \times 2$ crossover design. 
$\mathrm{C}_{\max }$ and area under the curve from time 0 to the last measurable concentration $\left(\mathrm{AUC}_{0 \text {-tlast }}\right)$ were considered as the primary target variables; area under the curve from time 0 to the infinite time $\left(\mathrm{AUC}_{0-[?]}\right)$, time to reach the peak concentration $\left(t_{\max }\right)$, terminal half life $\left(t_{1 / 2}\right)$, terminal disposition rate constant $\left(\lambda_{z}\right)$ and mean residence time (MRT) were declared as the secondary target variables in this bioequivalence study.

$\mathrm{C}_{\max }$ and $\mathrm{t}_{\max }$ for Favipiravir were obtained directly by plasma concentration-time curves. $\mathrm{AUC}_{0 \text {-tlast }}$ was calculated using the trapezoidal rule. $\mathrm{AUC}_{0-[?]}$ was calculated by summing $\mathrm{AUC}_{0 \text {-tlast }}$ and extrapolated area. The latter was determined by dividing the last measuredconcentration by $\lambda_{z}$ which was estimated byregression of the terminal log-linear plasma concentration time points.

$\mathrm{C}_{\max }$ and $\mathrm{AUC}_{0 \text {-tlast }}$ were tested for statistically significant differences by means of the Analysis of Variance (ANOVA) test procedure after logarithmic transformation (ln). The effects of ANOVA were treatment, period, sequence and subject within the sequence and tested at $5 \%$ level of significance.

In the assessment of bioequivalence, confidence intervals approach was used. The two one-sided hypothesis at the $5 \%$ level of significance were tested by constructing the $90 \%$ confidence intervals (90\% CIs) for the geometric mean ratios of test/reference products. The two formulations were considered as bioequivalent if the $90 \%$ CIs were within $80.00-125.00 \%$ for $\mathrm{C}_{\max }$ and $\mathrm{AUC}_{0 \text {-tlast }}$. Difference in $\mathrm{t}_{\max }$ was evaluated nonparametrically.

All statistical analysis were done using Phoenix WinNonlin (Version 8.1, Certara L.P.).

Also, ANOVA and determination of $90 \%$ CIs were applied to non-logaritmic transformed data of $\mathrm{t}_{\max }, \mathrm{t}_{1 / 2}$, $\lambda_{z}$ and MRT and to ln transformed data of $\mathrm{AUC}_{0-[?] \cdot}$.

\section{Results}

57 subjects were screened. 30 subjects were randomised and included into the study. The subjects were divided into two groups according to the randomisation table. There was one drop-out (Subject 05 didn't want to continue trial by his freewill at isolation days). As a result, 29 subjects completed the clinical phase of the study. All of the subjects were Caucasian. The mean \pm SD age of subjects is $25.45 \pm 3.86$ years and the mean \pm SD body mass index (BMI) was $26.12 \pm 2.10$. The demographic data of subjects are presented in Table 1 . There was no protocol deviation through the clinical period.

Table 1. Demographic data of the subjects.

\begin{tabular}{lllll}
\hline $\mathbf{n = 2 9}$ & Age (year) & Weight $(\mathrm{kg})$ & Height $(\mathbf{c m})$ & Body mass index \\
Mean & 25.45 & 79.52 & 174.45 & 26.12 \\
SD & 3.86 & 7.96 & 6.41 & 2.10 \\
Minimum & 20 & 68 & 161 & 23 \\
Maximum & 35 & 95 & 183 & 30 \\
\hline
\end{tabular}

Actual time of sampling was used in the estimation of the pharmacokinetic parameters.

In period II, there was observed no pre-dose drug concentrations, which indicates that the washout period of 48 hours was sufficient.

The pharmacokinetic parameters for test and reference products are summarised in Table $\mathbf{2}$, the geometric least square means, ratios and 90\% CIs are summarised in Table 3. Average plasma concentration-time curves and average ln plasma concentration-time curves of test and reference products for single dose of Favipiravir are displayed in Figure 1 and $\mathbf{2}$, respectively.

For Test and Reference products, the mean \pm sd of $\mathrm{C}_{\max }$ were found $5411.624 \pm 2025.680 \mathrm{ng} / \mathrm{mL}$ and $5002.171 \pm 1231.177 \mathrm{ng} / \mathrm{mL}$, and the mean \pm sd of $\mathrm{AUC}_{0 \text {-tlast }}$ were found $9641.989 \pm 2545.142 \mathrm{hr} . \mathrm{ng} / \mathrm{mL}$ and $9907.170 \pm 2423.528 \mathrm{hr} . \mathrm{ng} / \mathrm{mL}$, respectively (Table $\mathbf{2}$ ).

The primary target variables data demonstrate the bioequivalence of test and reference products with regard 
to $90 \% \mathrm{CI}$ for $\mathrm{C}_{\max }$ of $92.92-119.89$ and for $\mathrm{AUC}_{0 \text {-tlast }}$ of $94.00-99.77$, which are within acceptance limits (80.00-125.00\%) [4]. The geometric mean ratios were found as $105.55 \%$ and $96.84 \%$ for $\mathrm{C}_{\max }$ and $\mathrm{AUC}_{0 \text {-tlast }}$, respectively (Table 3 ).

For the secondary endpoint data, the median of $\mathrm{t}_{\max }$ for Test and Reference product were found $0.50 \mathrm{hr}$ and $0.75 \mathrm{hr}$, respectively and ranged from $0.17 \mathrm{hr}$ to $1.66 \mathrm{hr}$ for test product and $0.17 \mathrm{hr}$ to $2.5 \mathrm{hr}$ for reference product. Besides, the mean \pm sd of $\mathrm{t}_{1 / 2}$ for Test and Reference product were found $1.266 \pm 0.251 \mathrm{hr}$ (ranged from $0.867 \mathrm{hr}$ to $1.998 \mathrm{hr}$ ) and $1.319 \pm 0.245 \mathrm{hr}$ (ranged from $0.907 \mathrm{hr}$ to $2.072 \mathrm{hr}$ ), respectively. The mean $\pm \mathrm{sd}$ of $\lambda_{\mathrm{z}}$ for Test and Reference product were $0.566 \pm 0.0991 / \mathrm{hr}$ (ranged from $0.3471 / \mathrm{hr}$ to $0.7991 / \mathrm{hr}$ ) and $0.542 \pm 0.0941 / \mathrm{hr}$ (ranged from $0.3341 / \mathrm{hr}$ to $0.7641 / \mathrm{hr}$ ), respectively (Table 2 ).

Table 2. The arithmetic mean \pm sd of pharmacokinetic parameters of single oral dose of $200 \mathrm{mg}$ Favipiravir in test drug (Favicovir $200 \mathrm{mg}$ Film Tablet, Atabay Kimya San. ve Tic. A.Ş.-Turkey) and reference drug (Avigan $200 \mathrm{mg}$ Film Tablet, Toyama Chemical Industry Co.Ltd.-Japan) in healthy adult male subjects under fasting conditions. ${ }^{\mathrm{a}}$

\begin{tabular}{|c|c|c|}
\hline & Arithmetic Mean \pm SD $(n=29)$ & Arithmetic Mean \pm SD $(n=29)$ \\
\hline Parameters (Units) & Test $(\mathrm{T})$ & Reference (R) \\
\hline $\mathrm{C}_{\max }(\mathrm{ng} / \mathrm{mL})$ & $5411.624 \pm 2025.680$ & $5002.171 \pm 1231.177$ \\
\hline $\mathrm{AUC}_{0 \text {-tlast }}(\mathrm{ng} . \mathrm{hr} / \mathrm{mL})$ & $9641.989 \pm 2545.142$ & $9907.170 \pm 2423.528$ \\
\hline $\operatorname{AUC}_{0-[?]}(\mathrm{ng} \cdot \mathrm{hr} / \mathrm{mL})$ & $9910.494 \pm 2618.916$ & $10152.115 \pm 2507.694$ \\
\hline$t_{\max }(h r)$ & $0.50(0.17-1.66)$ & $0.75(0.17-2.5)$ \\
\hline$t_{1 / 2}(h r)$ & $1.266 \pm 0.251$ & $1.319 \pm 0.245$ \\
\hline$\lambda \zeta(1 / n \rho)$ & $0.566 \pm 0.099$ & $0.542 \pm 0.094$ \\
\hline MRT (hr) & $2.105 \pm 0.429$ & $2.161 \pm 0.428$ \\
\hline
\end{tabular}

${ }^{\mathrm{a}} t_{\text {max }}$ values are presented as median with range (minimum-maximum) in parentheses

\section{Statistical Parameters}

Table 3. Geometric Least Square Means, Ratio and 90\% Confidence Intervals of test drug (Favicovir 200 mg Film Tablet, Atabay Kimya San. ve Tic. A.Ş.-Turkey) and reference drug (Avigan 200 mg Film Tablet, Toyama Chemical Industry Co.Ltd.-Japan) in healthy adult male subjects under fasting conditions

\begin{tabular}{llllllll}
\hline Parameter & Difference & DiffSE & TESTLSM & REFLSM & Ratio\% & $90 \%$ CI & ISCV\% \\
\hline $\ln \left(\mathrm{C}_{\max }\right)$ & 0.0540 & 0.0748 & 8.5400 & 8.4860 & 1.0555 & $0.9292-1.1989$ & 29.053 \\
$\ln \left(\right.$ AUC $\left._{0 \text {-tlast }}\right)$ & -0.0321 & 0.0175 & 9.1435 & 9.1756 & 0.9684 & $0.9400-0.9977$ & 6.668 \\
$\ln \left(\mathrm{AUC}_{0-[?]}\right)$ & -0.0285 & 0.0171 & 9.1713 & 9.1998 & 0.9719 & $0.9440-1.0006$ & 6.511 \\
$\mathrm{t}_{\max }(\mathrm{hr})$ & -0.1330 & 0.1090 & 0.6053 & 0.7383 & 0.8199 & $0.5685-1.0714$ & \\
$\mathrm{t}_{\frac{1}{2}}(\mathrm{hr})$ & -0.0541 & 0.0198 & 1.2654 & 1.3195 & 0.9590 & $0.9334-0.9846$ & \\
$\lambda_{\mathrm{z}}(1 / \mathrm{hr})$ & 0.0247 & 0.0081 & 0.5662 & 0.5415 & 1.0456 & $1.0202-1.0711$ & \\
MRT $(\mathrm{hr})$ & -0.0636 & 0.0636 & 2.1018 & 2.1654 & 0.9706 & $0.9206-1.0207$ & \\
\hline
\end{tabular}




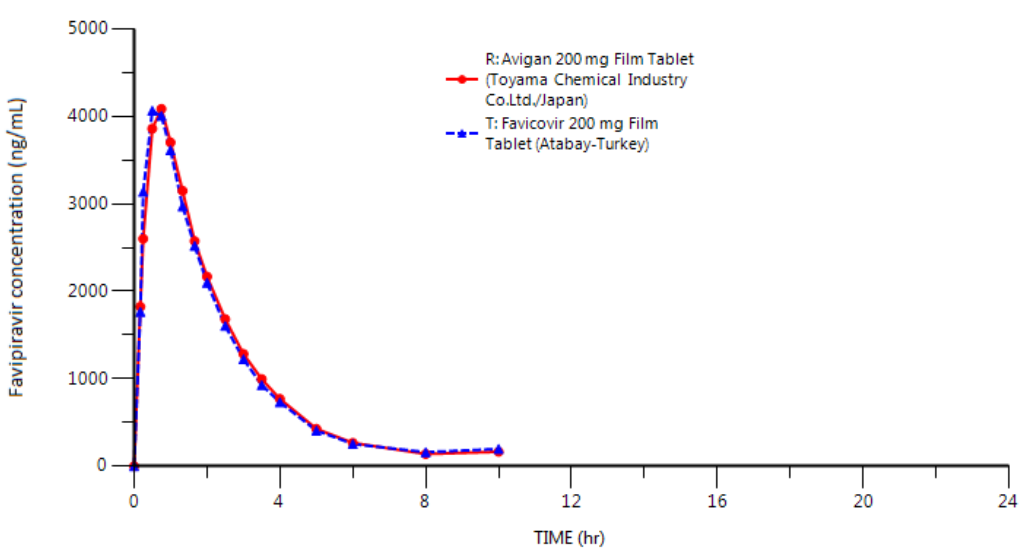

Figure 1: Mean plasma concentration-time curves after a single dose of a test drug (Favicovir $200 \mathrm{mg}$ Film Tablet, Atabay Kimya San. ve Tic. A.Ş.-Turkey) and a reference drug (Avigan $200 \mathrm{mg}$ Film Tablet, Toyama Chemical Industry Co.Ltd.-Japan) of oral Favipiravir in healthy adult male subjects $(\mathrm{n}=29)$ under fasting conditions.

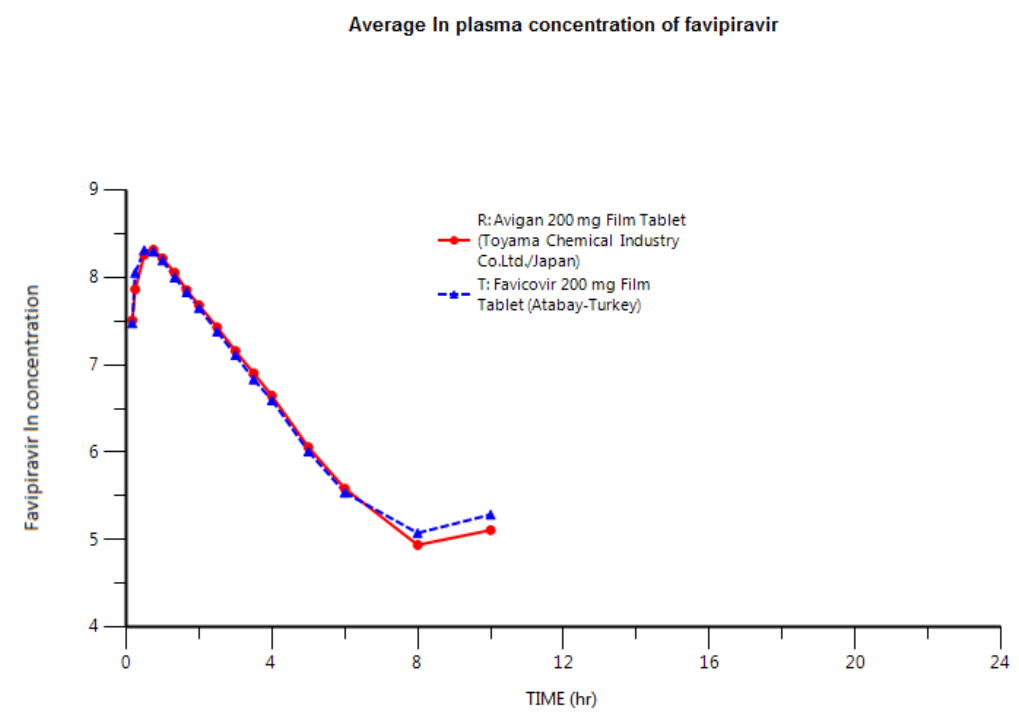

Figure 2: Average ln plasma concentration curves of Favipiravir after a single dose of a test drug (Favicovir $200 \mathrm{mg}$ Film Tablet, Atabay Kimya San. ve Tic. A.Ş.-Turkey) and a reference drug (Avigan $200 \mathrm{mg}$ Film Tablet, Toyama Chemical Industry Co.Ltd.-Japan) of oral Favipiravir in healthy adult male subjects $(\mathrm{n}=$ 29) under fasting conditions.

\section{Safety and Tolerability}


No adverse event occurred in all two periods. The overall tolerability of the products found to be good. There were no serious adverse events or adverse reactions reported throughout the study.

\section{Discussion}

Generic drugs are the cornerstones of pharmaceutical market. A novel generic formulation of Favipiravir, which is an antiviral compound with a wide range of antiviral activity against various influenza virus strains and is being used for Covid-19 treatment was developed and the pharmacokinetic properties were assessed in a bioequivalence study.

The ANOVA results showed that treatment, sequence, period and subject within sequence had no statistically significant effects on $\mathrm{C}_{\max }$ and $\mathrm{AUC}_{0 \text {-tlast }}$ (except subject within sequence effect for only $\mathrm{AUC}_{0 \text {-tlast }}$ ). Since the sequence or carry-over effect was not significant, ANOVA was valid.

Besides, ISCVs were found as $29.053 \%$ and $6.668 \%$ and the geometric mean ratios were found as $105.55 \%$ and $96.84 \%$ for $\mathrm{C}_{\max }$ and $\mathrm{AUC}_{0 \text {-tlast }}$, respectively.

$t_{\max }$ was analyzed using Mann-Whitney $\mathrm{U}$ test and there were not significant difference between two formulations with a significance level of $5 \%$ in regard to $t_{\max }(\mathrm{p}=0.321)$.

\section{Conclusions}

Since the $90 \%$ CIs for the test/reference geometric mean ratios for $\mathrm{C}_{\max }$ and $\mathrm{AUC}_{0 \text {-tlast }}$ of Favipiravir are contained within the acceptance limits preset in the Clinical Study Protocol, 80.00-125.00\%; according to the applied bioequivalence study, it is concluded that test and reference Favipiravir products are bioequivalent under fasting conditions and test product can be licensed under the requirements of regulatory authorities. Moreover, both study drugs were well-tolerated and considered to be safe.

\section{Acknowledgements}

This study was funded by Atabay Pharmaceutıcals and Fine Chemıcals Inc., Istanbul, Turkey. Clinical part of this study was conducted at Farmagen Good Clinical Practice Center.

\section{Data Availability}

The data that support the findings of this study are available from the corresponding author upon reasonable request. Some data may not be made available because of privacy or ethical restrictions.

\section{Conflict of Interest}

Authors declare no conflict of interest.

\section{References}

[1]. Favipiravir in Covid-19 International Journal of Progressive Sciences and Technologies (IJPSAT) Vol. 19 No. 2 March 2020, pp. 143-145

[2]. Report on the Deliberation Results of Avigan Tablet $200 \mathrm{mg}$ (Favipiravir), Pharmaceuticals and Medical Devices Agency (PMDA), 04.03.2014 https://www.pmda.go.jp/files/000210319.pdf

[3]. Cai, Q, Yang M, Liu D, Chen J, Shu D, Xia J et al. Experimental Treatment with Favipiravir for COVID- 19: An Open-Label Control Study. Engineering, in press, avaible online 16.04.2020.

[4]. Chen C, Zhang Y, Huang J, Yin P, Cheng Z, Wu J, Chen S, et al. Favipiravir versus Arbidol for COVID-19: A Randomized Clinical Trial. MedRxiv 2020

[5]. Guideline on The Investigation of Bioequivalence. CPMP/EWP/QWP/1401/98 Rev.1/Corr., London, EMA, 20 January 2010.

[6]. Guidance for Industry. Bioavailability and bioequivalence studies for orally administered drug productsGeneral Considerations. FDA, CDER, March 2014. 
[7]. The Guidance for GCP, published by the Ministry of Health of Turkey. Circular, 13.11.2015.

[8]. Guideline on Bioanalytical Method Validation, EMEA/CHMP/EWP/192217/2009 Rev.1 Corr.2, London, 21 July 2011. 